\title{
Refractory Kawasaki Disease: Infliximab or Methotrexate Therapy?
}

Sir,

We read with interest the article by Girish et al. ${ }^{1}$ They reported a 4-yr-old boy with refractory Kawasaki disease (KD), who was resistant to additional IVIG and methylprednisolone pulse therapy, but successfully treated with anti-TNF- $\alpha$ agent (infliximab). ${ }^{1}$

However, infliximab is very expensive to use as a second-line drug in refractory $\mathrm{KD}$ in developing countries. Therefore, one of our authors have previously used low-dose oral methotrexate as a treatment for patients with refractory KD. ${ }^{2}$ In his study, methotrexate treatment resulted in rapid defervescence, improvement in clinical symptoms, normalizationm of acute-phase reactants, and no progression of coronary artery dilatation in severe KD patients resistant to additional IVIG and intravenous dexamethasone. ${ }^{2}$ Methotrexate was also discontinued with no recurrence of fever in all patients. ${ }^{2}$ Because methotrexate is cheap, easy to administer (oral, weekly), easily available and less toxic than infliximab, it could effectively be used before applying infliximab to KD patients refractory to additional IVIG or high-dose steroid therapy.

Also, we should think about the responsiveness to infliximab in patients with KD. Although not studied in KD, Martínez-Borra et al. reported that high serum TNF$\alpha$ levels were associated with lack of response to infliximab in fistulizing Crohn's disease. ${ }^{3}$ Marotte et al. also demonstrated that the level of circulating TNF- $\alpha$ bioactivity was higher in good responders than in poor responders, although the TNF- $\alpha$ protein levels were not associated with the response to infliximab. ${ }^{4}$
In conclusion, because coronary artery aneurysm can develop within a relatively short time in young infants, low-dose oral methotrexate could be one of the useful drugs in refractory KD before considering infliximab. Further studies are also necessary to identify the factors predictive of the clinical response to infliximab in KD, and the relationship between the responsiveness to infliximab and the degree of increased serum TNF- $\alpha$ levels or circulating TNF- $\alpha$ bioactivity should be further elucidated in the future.

Jae Il Shin, Jae Seung Lee, Jae Young Choi, and Dong Soo Kim Department of Pediatrics, Yonsei University College of Medicine, Severance Children's Hospital, Seoul, Korea E-mail:dskim6634@yuhs.ac

\section{REFERENCES}

1. Girish M, Subramaniam G. Infliximab treatment in refractory Kawasaki syndrome. Indian J Pediatr 2008; 75: 521-522.

2. Ahn SY, Kim DS. Treatment of intravenous immunoglobulinresistant Kawasaki disease with methotrexate. Scand J Rheumatol 2005; 34: 136-139.

3. Martínez-Borra J, López-Larrea C, González S et al. High serum tumor necrosis factor-alpha levels are associated with lack of response to infliximab in fistulizing Crohn's disease. Am J Gastroenterol 2002; 97: 2350-2356.

4. Marotte H, Arnaud B, Diasparra J, Zrioual S, Miossec P. Association between the level of circulating bioactive tumor necrosis factor alpha and the tumor necrosis factor alpha gene polymorphism at -308 in patients with rheumatoid arthritis treated with a tumor necrosis factor alpha inhibitor. Arthritis Rheum 2008; 58: 1258-1263.

\section{Author's Reply}

We appreciate your comments on our article titled "Infliximab treatment in Refractory Kawasaki Disease". While all pedistricians all over the world continue to struggle with IVIG resistant KD, this topic has been discussed to engage the attention of researchers at many centers in the world. Hopefully soon pediatricians can look beyond isolated case reports or uncontrolled case series and be guided by randomized double-blind controlled studies. Your experience with methotrexate in IVIG resistant KD is indeed very interesting, but the small number and lack of control group make it difficult to draw any definite conclusions. Currently, a two-center, randomized, double-blind, placebo controlled trial is in progress in the U.S (ref Jane C. Burns. "Kawasaki Disease Update". Indian J Ped 2009;76:71-76.) to evaluate the role of Infliximab in IVIG resistant $\mathrm{KD}$.

Methotrexate could also be evaluated by you in a similar study, though at a conceptual level, I have my reservation about the use of a slowly acting antiinflammatory drug in this condition.

Drs Meenakshi Girish and Subramaniam Dept of Pediatrics, NKPSIMS Nagpur, India.E-mail:min_gir@rediffmail.com 